\title{
Non-invasive mechanical ventilation in acute respiratory failure due to chronic obstructive pulmonary disease: correlates for success
}

\author{
N Ambrosino, K Foglio, F Rubini, E Clini, S Nava, M Vitacca
}

\begin{abstract}
Background - Non-invasive mechanical ventilation is increasingly used in the treatment of acute respiratory failure in patients with chronic obstructive pulmonary disease (COPD). The aim of this study was to identify simple parameters to predict the success of this technique.

Methods - Fifty nine episodes of acute respiratory failure in 47 patients with COPD treated with non-invasive mechanical ventilation were analysed, considering each one as successful $(78 \%)$ or unsuccessful (22\%) according to survival and to the need for endotracheal intubation.
\end{abstract}

Results - Pneumonia was the cause of acute respiratory failure in $38 \%$ of the unsuccessful episodes but only in $9 \%$ of the successful ones. Success with non-invasive mechanical ventilation was associated with less severely abnormal baseline clinical and functional parameters, and with less severe levels of acidosis assessed during an initial trial of non-invasive mechanical ventilation.

Conclusions - The severity of the episode of acute respiratory failure as assessed by clinical and functional compromise, and the level of acidosis and hypercapnia during an initial trial of non-invasive mechanical ventilation, have an influence on the likelihood for success with non-invasive mechanical ventilation and may prove to be useful in deciding whether to continue with this treatment.

(Thorax 1995;50:755-757)

Keywords: non-invasive mechanical ventilation, acute respiratory failure, chronic obstructive pulmonary disease.

Non-invasive mechanical ventilation is increasingly being used in the treatment of acute respiratory failure in patients with chronic obstructive pulmonary disease (COPD). To date, controlled studies comparing non-invasive mechanical ventilation with endotracheal intubation are lacking, so the use of non-invasive mechanical ventilation as an alternative to intubation might, if unsuccessful, unduly delay endotracheal intubation. ${ }^{1-4}$ The aim of this study was to identify simple measures which could be used to predict whether patients with COPD could be successfully treated with noninvasive mechanical ventilation, and also to avoid unnecessary delay in intubation of those who deteriorate on non-invasive mechanical ventilation.

\section{Methods}

We retrospectively reviewed the data of 47 patients with COPD ( 31 men) undergoing 59 consecutive episodes of acute respiratory failure. All were chronically hypoxaemic and hypercapnic and on long term oxygen therapy. Patients with relevant concomitant diseases were excluded. All had undergone acute relapses of their primary disease and had been given non-invasive mechanical ventilation and met the following criteria: rapid deterioration in neurological status, ${ }^{5}$ acute onset of severe hypercapnia $\left(\mathrm{PaCO}_{2}>8.5 \mathrm{kPa}\right)$, acute decrease in $\mathrm{pH}(<7 \cdot 35)$, tachypnoea and/or abdominal paradox. The attending physicians considered that all these patients were likely to require mechanical ventilation and performed a short (1-2 hours) trial of non-invasive mechanical ventilation before endotracheal intubation when, according to their own clinical judgement, the clinical and functional status deteriorated despite non-invasive mechanical ventilation.

Mechanical ventilation was added to standard medical and oxygen therapy. Modalities of non-invasive mechanical ventilation were either pressure support ventilation (NPSV) (25 episodes) or intermittent positive pressure ventilation (NIPPV) in assisted/controlled mode (34 episodes) delivered through either nasal or facial masks. The ventilatory settings were as previously described. ${ }^{3}$ The following data were considered from the case records:

1. Last demographic and anthropometric data available before non-invasive mechanical ventilation: age, sex, weight $(\mathrm{kg}), \%$ of ideal body weight (\%IBW) measured with reference to the Metropolitan Life Insurance Company table ${ }^{6}$;

2. Last available serum levels of albumin;

3. Last functional characteristics recorded in stable state time before the acute respiratory failure (available in 42 patients) including spirometric parameters and arterial blood gas analysis breathing room air;

4. Causes of relapse including the following definitions: (a) pneumonia: the presence of lung infiltrates on the chest radiograph combined with any three of the following: fever, positive blood cultures, leucocytosis, or potential pathogenic bacterial cultures 
Table 1 Mean (SD) demographic, anthropometric, and clinical characteristics of patients and cause of acute respiratory failure according to outcome of non-invasive mechanical ventilation

\begin{tabular}{lccl}
\hline & $\begin{array}{c}\text { Group } 1 \\
(n=46)\end{array}$ & $\begin{array}{c}\text { Group } 2 \\
(n=13)\end{array}$ & p value \\
\hline Death (n) & 0 & 4 & \\
Age (years) & $64(8)$ & $68(8)$ & NS \\
Weight (kg) & $69(19)$ & $48(13)$ & $<0.01$ \\
IBW (\%) & $118(35)$ & $86(12)$ & $<0.05$ \\
Albumin (g/dl) & $3.8(0.5)$ & $3.4(0.4)$ & $\mathrm{NS}$ \\
Pneumonia & $4(8 \cdot 7 \%)$ & $5(38.5 \%)$ & \\
Exacerbation & $39(84.8 \%)$ & $8(61.5 \%)$ & \\
Pulmonary embolism & $3(6.5 \%)$ & $3.9(0.7)$ & $<0.00001$ \\
Neurological status score & $1.9(0.9)$ & $24(4)$ & $<0.0001$ \\
APACHE II & $18(4)$ & $3.0(1.5)$ & $<0.05$ \\
Compliance score & $3.7(0.8)$ & & \\
\hline
\end{tabular}

Table 2 Mean (SD) heart rate and blood gas levels at baseline and during non-invasive mechanical ventilation (NMV)

\begin{tabular}{|c|c|c|c|c|}
\hline & \multicolumn{2}{|l|}{ Group 1} & \multicolumn{2}{|l|}{ Group 2} \\
\hline & Baseline & $N M V$ & Baseline & $N M V$ \\
\hline $\begin{array}{l}\text { Heart rate (bpm) } \\
\text { pH } \\
\mathrm{PaCO}_{2}(\mathrm{kPa}) \\
\mathrm{PaO}_{2} / \mathrm{FIO}_{2}\end{array}$ & $\begin{array}{c}108(23)^{*} \\
7 \cdot 28(0.04)^{*} \\
10 \cdot 5(1 \cdot 7)^{*} \\
1.70(0.61)\end{array}$ & $\begin{array}{l}102(14) \\
7 \cdot 34(0.04) \dagger \\
8 \cdot 8(1.3) \dagger \\
2 \cdot 24(0.59)\end{array}$ & $\begin{array}{c}146(17) \\
7 \cdot 22(0 \cdot 08) \\
13 \cdot 1(3 \cdot 1) \\
1 \cdot 72(1 \cdot 0)\end{array}$ & $\begin{array}{c}138(34)^{* *} \\
7 \cdot 25(0.08)^{* *} \$ \\
11 \cdot 4(1 \cdot 6)^{* *} \\
2.0(0.87) \oint\end{array}$ \\
\hline
\end{tabular}

$t p<0.00001$ for difference between baseline and NMV.

$\mathrm{p}<0.05$ for difference between baseline and NMV.

p $<0.005$ for differences between baseline in groups 1 and 2 .

** $p<0.005$ for differences between blood gas levels during NMV in groups 1 and 2 .

from sputum; (b) exacerbation of COPD: increased dyspnoea with no other obvious cause for respiratory deterioration; (c) pulmonary embolism when confirmed by a perfusion and ventilation lung scan.

5. APACHE II score ${ }^{7}$ at the moment of starting non-invasive mechanical ventilation;

6. Neurological status assessed retrospectively from the case notes using a score proposed by Kelly and Matthay;

7. Subjective compliance to ventilation assessed by the physician in charge using an arbitrary score $(1=$ bad; $2=$ insufficient; $3=$ sufficient; $4=$ good; $5=$ very good $)^{3}$;

8. Arterial blood gas tensions and heart rate assessed immediately before (baseline) and during an initial 1-2 hour trial of noninvasive mechanical ventilation. Baseline arterial blood gas tensions were assessed with all patients breathing oxygen at an $\mathrm{FIO}_{2}$ set initially by the physician in charge.

Non-invasive mechanical ventilation was considered successful when patients reached levels of $\mathrm{pH}>7.35$ during spontaneous breathing without further worsening of neurological signs, and with improvement in tachypnoea and in abdominal paradox for at least $\mathbf{4 8}$ hours (group 1). Failure of non-invasive mechanical ventilation (group 2) was defined by the need for endotracheal intubation according to the judgement of the physician in charge or death during non-invasive mechanical ventilation.

DATA ANALYSIS

Comparison of differences of successful versus unsuccessful treatment was performed using a Student unpaired $t$ test with $p<0.05$ being considered significant. Comparison of baseline data with those recorded during non-invasive mechanical ventilation was performed using a paired $t$ test, a $\mathrm{p}$ value of $<0.05$ being considered significant. The predictive models were developed using discriminant analysis. All the previously described variables were tested in an attempt to establish differences between the successful and unsuccessful group. Single predictor variable values were computed, treating each variable as though it was the only predictor available. Finally, these variables were tested together with a stepwise logistic regression analysis to assess which of them best predicted the results of the treatment in this multivariable context.

\section{Results}

The causes of acute respiratory failure, demographic, anthropometric, and clinical status of the patients are shown in table 1 .

Non-invasive mechanical ventilation was successful in 46 episodes of acute respiratory failure $(78 \%)$. The overall mortality was $8.5 \%$ (four of 47 patients). Three patients died during non-invasive mechanical ventilation, endotracheal intubation not having been offered. In one of them the cause of acute respiratory failure was pneumonia. Of the 10 patients undergoing endotracheal intubation one further patient with pneumonia died one week later.

The most frequent cause of acute respiratory failure in all patients was an exacerbation of COPD without clinical or radiological signs of pneumonia. Clinical, radiological, and laboratory evidence of pneumonia was found in $38.5 \%$ of episodes of acute respiratory failure unsuccessfully treated with non-invasive mechanical ventilation, but only in $8.7 \%$ of those successfully treated. Patients in whom noninvasive mechanical ventilation was unsuccessful were significantly underweight in comparison with those in whom non-invasive mechanical ventilation was successful. Noninvasive mechanical ventilation was also unsuccessful in patients with a significantly greater level of neurological deterioration, a higher APACHE II score, and a reduced level of compliance.

Arterial blood gas tensions and heart rate (available only in 34 patients in group 1 and seven in group 2) before and during the initial trial of non-invasive mechanical ventilation are shown in table 2. Patients in group 2 showed significantly more abnormal levels of baseline $\mathrm{pH}$ and $\mathrm{PaCO}_{2}$ and a significantly higher heart rate than those in group 1. The level of oxygenation as assessed by $\mathrm{PaO}_{2}, \mathrm{SaO}_{2}$, and $\mathrm{PaO}_{2} / \mathrm{FrO}_{2}$ was similar in the two groups. Noninvasive mechanical ventilation significantly improved $\mathrm{pH}$ and $\mathrm{PaCO}_{2}$ in both groups (mean reduction in $\mathrm{PaCO}_{2}$ of $15 \%$ and $10 \%$ in groups 1 and 2, respectively); nevertheless, even during non-invasive mechanical ventilation $\mathrm{pH}$ and $\mathrm{PaCO}_{2}$ of patients in group 2 remained severely compromised.

The last pulmonary function tests performed when stable before acute respiratory failure were available for only 37 patients of group 1 and five of group 2. Analysis showed that the only significant difference was found in forced vital capacity $(1.6(0.6) 1$ and $0.9(0.1) 1$ for 
Table 3 Accuracy of the single indexes used to predict outcome of non-invasive mechanical ventilation (NMV)

\begin{tabular}{llllll}
\hline & Predictability & Sensitivity & Specificity & $\begin{array}{l}\text { Positive } \\
\text { predictive } \\
\text { value }\end{array}$ & $\begin{array}{l}\text { Negative } \\
\text { predictive } \\
\text { value }\end{array}$ \\
\hline Body weight & 0.66 & 0.64 & 0.75 & 0.93 & 0.30 \\
\%IBW & 0.65 & 0.60 & 0.86 & 0.96 & 0.29 \\
Neurological status score & 0.84 & 0.80 & 1 & 1 & 0.57 \\
APACHE II score & 0.85 & 0.90 & 0.67 & 0.90 & 0.67 \\
Compliance score & 0.66 & 0.67 & 0.61 & 0.86 & 0.35 \\
Baseline Paco $\mathrm{PaCO}_{2}$ & 0.75 & 0.76 & 0.69 & 0.90 & 0.45 \\
NMV Paco & 0.85 & 0.87 & 0.73 & 0.93 & 0.57 \\
Baseline pH & 0.80 & 0.87 & 0.54 & 0.87 & 0.54 \\
NMV pH & 0.92 & 0.93 & 0.82 & 0.95 & 0.75 \\
\hline
\end{tabular}

groups 1 and 2, respectively; $\mathrm{p}<0 \cdot 05$ ). Because of the small number of cases with this variable, neither forced vital capacity nor heart rate were processed further.

The accuracy of the indices used to predict the success of non-invasive mechanical ventilation is illustrated in table 3 . The discriminant analysis showed that five of the variables considered had a predictive value $>0.80$ and were important in allowing a distinction between patients who were successfully treated and those who were not.

Nevertheless, the logistic regression analysis demonstrated that, when these variables were tested together, only baseline $\mathrm{pH}$ maintained a significant predictive effect, indicating that all other variables were in some way dependent on it. By this analysis baseline $\mathrm{pH}$ showed a sensitivity of $97 \%$ and a specificity of $71 \%$.

\section{Discussion}

This retrospective study shows that unsuccessful non-invasive mechanical ventilation was associated with pneumonia, reduced compliance with treatment, and with having a more severe clinical and functional condition. The response to an initial trial of non-invasive mechanical ventilation was also an important predictor of success.

In a previous report we have shown that non-invasive mechanical ventilation induced a significant reduction in the need for endotracheal intubation compared with an historical control group treated medically. ${ }^{3}$ The success rate of $78 \%$ and the mortality rate of $8.5 \%$ in this study are in agreement with previous studies. ${ }^{1-4}$

The response to non-invasive mechanical ventilation could be predicted by the level of acidosis before initiating ventilation. Non-invasive mechanical ventilation was effective in improving $\mathrm{PaCO}_{2}$ and $\mathrm{pH}$ in both groups of patients. Nevertheless, the absolute level of $\mathrm{PaCO}_{2}$ and $\mathrm{pH}$ after the initial trial of noninvasive mechanical ventilation accurately identified those patients who could be successfully ventilated. Our study confirms and extends a previous observation by Meduri et al on a larger number of patients with COPD. ${ }^{2}$ The use of arterial blood gas tensions measured at different levels of $\mathrm{FIO}_{2}$ may be criticised. Nevertheless, in this retrospective study we recorded the last available arterial blood gas tension at the moment the doctor decided on non-invasive mechanical ventilation. The level of $\mathrm{PaO}_{2}, \mathrm{SaO}_{2}$, and $\mathrm{PaO}_{2} / \mathrm{FIO}_{2}$ did not differ between successful and unsuccessful cases, so we are confident that the level of oxygenation did not influence the values in $\mathrm{PaCO}_{2}$ of the two groups.

The incidence of the causes of acute respiratory failure in this study is similar to previously reported data. ${ }^{8}$ Pneumonia was associated with most unsuccessful episodes. The occurrence of nosocomial pneumonia requiring admission to an ICU is associated with an increased risk of fatalities. ${ }^{9}$

Unsuccessful non-invasive mechanical ventilation was associated with reduced body weight expressed both as absolute and as \% IBW. The association between malnutrition and COPD has been recognised for many years. Factors related to nutritional status are considered as an independent influence on the course of COPD and, in a previous study, we found $\%$ IBW to be related to ICU admission. ${ }^{1011}$

Failure of non-invasive mechanical ventilation was associated also with a worse neurological status and reduced compliance with treatment. The importance of a compromised neurological status is easy to understand in view of the need for cooperation required to perform non-invasive mechanical ventilation.

In conclusion, the severity of the episode of acute respiratory failure as assessed by clinical and functional compromise and the level of acidosis and hypercapnia during an initial trial of non-invasive mechanical ventilation have an influence on the likelihood of success with noninvasive mechanical ventilation, and may prove useful in deciding the duration of a trial of this treatment. The results suggest that noninvasive mechanical ventilation should be instituted early in every patient before a severe acidosis ensues.

1 Brochard L, Isabey D, Piquet J, Amaro P, Mancebo J, Messadi AA, et al. Reversal of acute exacerbation of chronic obstructive lung disease by inspiratory assistance chronic obstructive lung disease by inspiratory assistanc

2 Meduri GU, Abou-Shala N, Fox RC, Jones CB, Leeper KV, Wunderink RG. Noninvasive face mask mechanical $\mathrm{KV}$, Wunderink RG. Noninvasive face mask mechanical
ventilation in patients with acute hypercapnic respiratory ventilation in patients with acute
failure. Chest 1991;100:445-54.

3 Vitacca M, Rubini F, Foglio K, Scalvini S, Nava S, Ambrosino N. Non-invasive modalities of positive pressure ventilation improve the outcome of acute exacerbations in COLD patients. Intensive Care Med 1993;19:450-5.

4 Bott J, Carroll MP. Conway JH, Keilty SEJ, Ward EM Brown AM, et al. Randomized controlled trial of nasal ventilation in acute ventilatory failure due to chronic obstructive airways disentilatory failure due to chron

5 Kelly BJ, Matthay MA. Prevalence and severity of neurologic dysfunction in critically ill patients. Influence on need for dysfunction in critically ill patients. Influence on need for 6 Metropolitan Life Insurance Company. Statistical bulletin. Metropolitan
1977;58:5.

7 Knaus WA, Draper EA, Wagner DP, Zimmerman JE. Apache II: a severity of disease classification system. Crit Care Med 1985;13:818-29.

8 Derenne JP, Fleury B, Pariente R. Acute respiratory failure of chronic obstructive pulmonary disease. Am Rev Respir Dis 1988;138:1006-33.

9 Potgieter PD, Hammond JMJ. Etiology and diagnosis of pneumonia requiring MICU admission. Chest 1992;101: 199-203.

10 Wilson DO, Rogers RM, Hoffman RM. Nutrition and chronic lung disease. Am Rev Respir Dis 1985;132:134765.

11 Vitacca M, Foglio K, Scalvini S, Marangoni S, Quadri A, Ambrosino N. Time course of pulmonary function before admission into ICU. A two year retrospective study of COLD patients with hypercapnia. Chest 1992;102:1737- 